\section{Another Place for Digging}

The fossil remains of three specimens of early Homo sapiens (see this issue, pages 1132-1138) which were discovered in the Omo River region of Ethiopia in 1967, together with the remains of a fourth specimen, which have yet to be described, have shed new light on one of the outstanding questions of palaeoanthropology - the origins of Homo sapiens. Before the discovery of these Omo fossils the fossil record of early Homo sapiens was extremely scant and fragmentary. Only three fossils were known-the Swanscombe, Steinheim and Fonte Chevade men. And none of them provided any real clue to the relationship between Homo sapiens of the Upper Pleistocene and the earlier Homo erectus of the Middle Pleistocene or the still earlier Lower Pleistocene, australopithecines and Homo habitis.

The Omo fossils were found by the Kenya Group, led by Richard Leakey, of the International Palaeontological Research Expedition to the Omo Valley, in a series of sediments known as the Kibish formation. The associated animal fossils, which included remains of buffalo, recdbucks and elephants, and the few stone tools associated with the human remains, provide little evidence of the age of the three Homo sapiens although they should yield valuable evidence of the local environment. But the detailed stratigraphic analysis of the hominid sites reported by Professor Karl Butzer, a membər of the US group of the expedition (page 1133 ), indicates that the three fossils are at least 35,000 years old and probably a great deal older. Dating remains older than the effective range of the carbon-14 method is necessarily an uncertain business, but some of the people who have closely examined the material believe it could be as old as 60,000 years and perhaps, though much less likely, 100,000 years old.

Whatever their absolute age, two of the hominid fossils, Omo I and II, are contemporaneous and older than Omo III. In their African context, Omo I and II are either late Middle Pleistocene or early Late Pleistocene forms, which is one of the reasons why they are so important; they push the African history of Homo sapiens a long way back into the Pleistocene. The other intriguing feature of the Omo I and II skulls is the striking difference in their shape. Even a cursory glance at the photographs of the backs of the two skulls (see page 1137) shows that whereas Omo I tapers at the neck, and is essentially indistinguishable from the skull of a modern man, the post cranial structure of Omo II is comparatively broad.

What does this mean? Obviously, as Dr M. H. Day says, it shows that the range of normal variation of bone structure in populations of early Homo sapiens was every bit as wide as those known from the remains of later sapients dug up in other parts of the world.
Secondly, the broad based skull of Omo II is a characteristic feature of the Middle Pleistocene Homo erectus, although Omo II is undoubtedly a sapient, because its cranial capacity is $1,400 \mathrm{ml}$., and the usual range for Homo erectus is about 800 to $1,100 \mathrm{ml}$. It could well be, therefore, that Omo II is an example of a link between Homo erectus and Homo sapiens. This emphasizes how lucky it was that Omo I and Omo II were found close together with evidence indicating that they were indeed contemporary. Had they been found separately it would have been natural enough, on the basis of their morphology, to suggest that Omo II was an ancestor rather than contemporary of Omo I.

It is, of course, premature on the evidence of these two skulls to suggest that Homo sapiens certainly evolved from Homo erectus. Omo II also shares some characteristics with the habilines Solo man, which was found in Java, and Rhodesian man, and Dr Day's cryptic final sentence mentions the possibility of "the origin of Homo sapiens in the Lower Middle Pleistocene or even the Lower Pleistocene periods". It may be that both Homo erectus and Homo sapiens evolved independently and parallel in time from populations of Lower Pleistocene Homo habilis. The discovery of such early remains of Homo sapiens in Africa certainly increases the credibility of this idea, although it comes nowhere near proving it.

\section{PHYSICS \\ Moral Support for Einstein}

Physicists hunting for gravitational waves have at times seemed to resemble astronomers looking for neutron stars, or even zoologists probing the depths of Loch Ness: lots of false alarms, and a lingering suspicion that the whole scheme is rather crackpot. But now the good news from the United States is that the faith of one man in particular, Professor Joseph Weber of the University of Maryland, may have been vindicated. In Monday's Physical Review Letters (22, $1320 ; 1969)$, Weber says that, since the end of December last year, there have been seventeen occasions when disturbances which seem to be gravitational waves have swept through detectors at the Argonne National Laboratory, Illinois, and at the University of Maryland simultaneously. The point is that the chances of the coincidences occurring by accident is "incredibly small", that the two detectors $1,000 \mathrm{~km}$ apart must have been excited by the same disturbances and that the only disturbances which could be responsible are gravitational waves.

It has been a long haul for Weber and his colleagues. Work has been going on throughout the $1960 \mathrm{~s}$, using piezoelectric crystals bonded to aluminium cylinders to pick up the vibrations caused by gravitational waves. Already coincidences have been observed in the outputs 